\title{
Editorial
}

\section{Temporal dynamics of content communication}

Scientometrics has experienced exponential growth in terms of number of papers submitted to area journals, and has also become a permanent source for advice and strategic decision-making for researchers, policy-makers, research managers and others. However, the most relevant effect may be the number of indicators that have been developed in order to account for the dynamics of research communication and the output of academic communities.

At the same time, validity and utility of indicators have increased in societies both in the first and third worlds, an issue that has been dealt with in several editorials in Universitas Psychologica, given that our communities can be affected by the way knowledge is used, communicated and applied. It is not a coincidence that this discussion has also been taken to high impact journals (Science, Nature, Neuron), where it has met both favourable and unfavourable reactions.

Some of these indicators are associated to how research communities use publications. Specifically, times and publication schedules have become relevant indicators, especially in Latin America, a region that has experienced tremendous growth in article output in all areas, more so than any other region.

This growth allows us to analyse indicators to compare Latin America to other regions or country-to-country comparisons, controlling for similar socioeconomic conditions, such as investment, education, skills and relationships, and also knowledge and information infrastructures. The development of these measurements has been high enough that new indicators have been proposed to predict the potential impact of a new publication. This would show that interpretation of these indicators is complex and involves more relevant, less local information.

It is clear, however, that some of these indicators are important, especially those related to publication schedules. The temporal dimension is more important today than ever due to institutional demands, knowledge obsolescence and the need for cooperation networks, but also because of the growing awareness that results should be made available quickly.

This is why preprint articles and DOI (Digital Object Identifiers) will be included in Universitas Psychologica from this issue (volume 12, issue 3, 2013), to promote faster citation. We expect to enter a new development phase by making papers available before the print edition. This alternative follows a worldwide publication trend, and shows that our emergent academic communities have started to create, communicate and appreciate their own knowledge. This may be why the hegemony of traditional impact indicators has been questioned.

We have also noticed, upon analysing tables of most-read and most-downloaded articles in our journal, that it is articles from the past few years that have a higher download rate, which suggests a clear trend in the use of knowledge. 
The growth experienced by our journal and its inclusion in the first quartiles in large information systems have involved changes and challenges, in terms of quality, quantity and prestige. This is why we feel to need to point out that we are constantly in a path towards advancement and we expect to be able to add new features to the website, in our quest for shorter publication times and higher quality of contents.

WILSON LÓPEZ LÓPEZ

EDITOR 


\section{Editorial}

\section{Las dinámicas temporales de la comunicación de contenidos}

La cienciometría ha tenido un desarrollo exponencial no solo en los trabajos que se presentan a revistas del área como en revistas especializadas sino que se ha convertido en una fuente permanente de consulta y de direccionamiento estratégico para investigadores y actores decisores y gestores de investigación y otras esferas sociales. Pero lo más relevante, tal vez sea, la multiplicidad de indicadores que han aparecido para dar cuenta de las dinámicas de la comunicación de la investigación y en general de la producción de las comunidades académicas.

De forma concurrente, $y$ ha sido objeto de varias editoriales de Universitas Psychologica, el tema de la validez y utilidad de los indicadores aumenta el interés al interior de las sociedades, tanto de primer como de tercer mundo, que pueden verse influenciadas por la forma como es utilizado, difundido y aplicado el conocimiento generado y publicado. No en vano esta discusión ha sido llevada a las revistas tradicionalmente denominadas de alto impacto (Science, Nature, Cell, Neuron) generando reacciones a favor y en contra del uso de estos indicadores de cienciometría.

Algunos de estos indicadores están asociados a los usos que la comunidad de investigadores hacen de la producción publicada en revistas. En especial, los tiempos de estos usos se han convertido en un indicador muy relevante, esto ante la creciente explosión de revistas y artículos en América Latina que ha tenido un crecimiento superior al mundo en la producción de artículos en todas las áreas.
Este crecimiento hoy permite que podamos analizar indicadores que comparan la región contra la región o entre países que tienen condiciones similares de tipo socioeconómico en términos de inversión de recursos económicos, formación, capacidades y relaciones de los investigadores, como de las infraestructuras de conocimiento e información. Además, ha sido tal el interés en el desarrollo de estas mediciones que se han propuesto indicadores para predecir incluso el impacto que tendría una publicación. Esto mostraría que cada vez la forma de interpretación de estos indicadores es más compleja e implica información más relevante y menos local.

Sin embargo, es claro que algunos de estos indicadores resultan críticos en especial los que tienen que ver con el tiempo de la publicación, la dimensión temporal hoy cobra más sentido para los investigadores por las exigencias institucionales, la vigencia del conocimiento y la necesidad de generar redes de cooperación, pero también por la creciente conciencia de la importancia de hacer visible rápidamente los resultados de las investigaciones.

Esta es la razón por la cual a partir de este nuevo número de la revista (volumen 12, número 3 de 2013) que se incluirá la disposición de los artículos en forma preprint con su correspondiente número de (identificación de objetos digitales) DOI con el cual puede ser citado más rápidamente. Esperamos entrar en una nueva fase de desarrollo al poner a disposición general los artículos antes de estar en su versión de diagramación final. Esta alternativa, entre otros factores, obedece a una dinámica que 
intenta ir a la par de la gran industria editorial a nivel mundial, mostrando el desarrollo de comunidades académicas emergentes en sociedades que han comenzado a producir, difundir y apreciar su propio conocimiento. Quizás por esta razón los indicadores tradicionales de medición de impacto se han visto vulnerados en su hegemonía por el surgimiento de nuevas comunidades académicas en desarrollo.

Adicionalmente, al realizar así un análisis de los artículos que más son consultados y descargados en nuestra revista encontramos que son los artículos de los últimos años y no los que han sido publicados con anterioridad, los que se encuentran con un mayor tráfico de descarga, sugiriendo una tendencia en la consulta y consumo de información reciente.

El crecimiento de Universitas Psychologica, la clasificación en los primeros cuartiles en grandes sistemas de información han implicado muchos cambios y desafíos. Ha sido un reto constante, concomitante con el crecimiento de la publicación, en términos de calidad, cantidad y prestigio. Por esto podemos señalar que constantemente nuestra revista está en proceso de avance y esperamos poder incorporar nuevas mejoras en web en las que estamos trabajando, buscando no solo mejorar los tiempos si no el acceso y la calidad de los artículos.

\section{WILSON LÓPEZ LÓPEZ}

EDITOR 